\title{
Coexistence of Local Moment Magnetism and Heavy-Fermion Superconductivity in $\mathrm{UPd}_{2} \mathrm{Al}_{3}$
}

\author{
R. Feyerherm, ${ }^{1}$ A. Amato, ${ }^{1}$ F. N. Gygax, ${ }^{1}$ A. Schenck, ${ }^{1}$ C. Geibel, ${ }^{2}$ F. Steglich, ${ }^{2}$ N. Sato,${ }^{3}$ and T. Komatsubara ${ }^{3}$ \\ ${ }^{1}$ Institute for Particle Physics, Eidgenössische Technische Hochschule Zürich, CH-5232 Villigen PSI, Switzerland \\ ${ }^{2}$ Institut für Festkörperphysik, Technische Hochschule Darmstadt, D-64289, Darmstadt, Germany \\ ${ }^{3}$ Department of Physics, Tohoku University, Sendai 980, Japan \\ (Received 14 March 1994)
}

\begin{abstract}
We report muon spin rotation measurements on the antiferromagnetic heavy-fermion superconductor $\mathrm{UPd}_{2} \mathrm{Al}_{3}$ in the superconducting state. The London penetration depth is found to be approximately isotropic, $\lambda_{\perp}(0)=4800 \pm 500 \AA$ and $\lambda_{\|}(0)=4500 \pm 500 \AA$. The $\mu^{+}$Knight shift behavior below $T_{c}$ indicates that local moment magnetism and superconductivity are carried by different electron substrates of $5 f$ character, one of which involves the heavy quasiparticles. For the latter a nearly isotropic magnetic susceptibility $\chi=1.7 \times 10^{-3} \mathrm{emu} / \mathrm{mole}$ can be estimated.

PACS numbers: 74.70.Tx, 75.30.Mb, 76.75. $+\mathrm{i}$
\end{abstract}

The large variations in the ground state properties of the six known heavy-fermion (HF) superconductors has to date prevented the development of a consistent microscopic theory of HF superconductivity [1,2]. A prominent puzzle is the coexistence of superconductivity and antiferromagnetic (AFM) ordering in most of these compounds, believed to arise from the same set of $f$ electrons. While for $\mathrm{CeCu}_{2} \mathrm{Si}_{2}$ there is evidence that both phenomena are in competition [3-5], they appear to coexist homogeneously in the U-based systems. In $\mathrm{UPt}_{3}$ [6], $\mathrm{U}_{1-x} \mathrm{Th}_{x} \mathrm{Be}_{13}$ [7], and $\mathrm{URu}_{2} \mathrm{Si}_{2}$ [8] very small ordered moments of the order of $\left(10^{-3}-10^{-2}\right) \mu_{B} / \mathrm{U}$ atom have been observed. In contrast, the recently discovered HF superconductors $\mathrm{UNi}_{2} \mathrm{Al}_{3}$ [9] and $\mathrm{UPd}_{2} \mathrm{Al}_{3}$ [10] exhibit relatively large ordered moments $\left[(0.12-0.24) \mu_{B}\right.$ $[11,12]$ and $0.85 \mu_{B}$ [13], respectively]. The simple AFM structure of $\mathrm{UPd}_{2} \mathrm{Al}_{3}\left(T_{N}=14 \mathrm{~K}[10,13]\right)$ is believed to be carried by local moments [14]. Moreover, in the latter two compounds, as well as in $\mathrm{URu}_{2} \mathrm{Si}_{2}$, the AFM ordering, established well above $T_{c}$, is essentially not affected by the superconducting transition $[8,11,15]$. This fact is intriguing in view of the unquestionable $5 f$ character of both the heavy quasiparticles forming the Cooper pairs and the electrons forming the AFM state. In the present Letter we wish to address this problem. On the basis of our results we will argue that in $\mathrm{UPd}_{2} \mathrm{Al}_{3}$ superconductiviy and magnetism behave as arising from different electron substates of $5 f$ character.

The present transverse field (TF) muon spin rotation ( $\mu$ SR) measurements have been carried out on single crystalline samples that were synthesized and characterized as reported previously [16] and that exhibit $T_{c}=$ $1.7 \mathrm{~K}$. The $\mu \mathrm{SR}$ data have been recorded at the low temperature $\mu$ SR facility and the general purpose $\mu$ SR spectrometer at the Paul Scherrer Institute. From measurements of the anisotropies of the $\mu^{+}$Knight shift and depolarization in the normal state (not shown) we unambiguously determined that all implanted $\mu^{+}$stop at the interstitial $b$ site $\left(\begin{array}{lll}0 & 0 & 1 / 2\end{array}\right)$ in the hexagonal unit cell, in agreement with earlier conclusions [17]. Only at this symmetric site the internal fields produced by the magnetic sublattices in the AFM ordered state cancel and thus no spontaneous $\mu^{+}$Larmor precession is observed. This particular feature allowed us to study the $\mu^{+}$Knight shift and TF $\mu^{+}$depolarization also below $T_{N}$. The measurements have been carried out (on field cooling) in fields of $H_{\text {ext }}=350 \mathrm{Oe}, 5 \mathrm{kOe}$, and $10 \mathrm{kOe}$ for the two principal orientations $\vec{H}_{\text {ext }} \| c$ and $\vec{H}_{\text {ext }} \perp c$, covering the temperature range $25 \mathrm{mK} \leq T \leq 2.5 \mathrm{~K}$ with the aim of studying the temperature and orientation dependence of the London penetration depth $\lambda$ and the $\mu^{+}$Knight shift in the superconducting state. In the following we will first describe the evaluation of these two parameters from the data and then discuss the experimental results.

A TF $\mu$ SR measurement of the time dependence of the muon polarization $G(t)$ allows one to determine the shift and the distribution of the $\mu^{+}$Larmor precession frequency and thus of the internal magnetic field $B$ at the $\mu^{+}$site. In an external magnetic field $H_{\text {ext }}>H_{c 1}$ the formation of the flux line lattice (FLL) in type-II superconductors produces inhomogeneities of both the superconducting order parameter $\psi$ and the magnetic field. The latter will give rise to an additional depolarization of the $\mu^{+}$ precession signal. From previous measurements of the temperature dependence of the TF $\mu^{+}$depolarization rates on a polycrystalline sample [17], it is known that between $T_{c}$ and $T_{N}$ an enhanced $\mu^{+}$depolarization is primarily caused by small internal fields produced by distortions of the magnetic sublattices and it is best described by an exponential $\exp (-\Lambda t)$. It has been proven by zero field $\mu$ SR that the internal fields do not change below $T_{c}$ and are present in the entire sample volume $[17,18]$. This indicates that the magnetism is not affected by the onset of superconductivity, but coexists with superconductivity on a microscopic scale below $T_{c}$, consistent with neutron scattering results [15]. Since the two sources of the field inhomogeneity below $T_{c}$ are of independent origin we may describe the time dependence of the $\mu^{+}$polarization $G(t)$ by a two channel expression,

$$
G(t)=\exp \left(-\Lambda_{0} t\right) G_{\mathrm{FLL}}(t),
$$


where $\Lambda_{0}$ is the saturation value of $\Lambda$ above $T_{c}$ [19] and $G_{\mathrm{FLL}}(t)$ corresponds to the Fourier transform of the magnetic field distribution $n(B)$. The second moment $\left\langle\Delta B^{2}\right\rangle$ of $n(B)$ as a function of $\lambda$ and $H_{\text {ext }}$ can be calculated from a modified London model that includes also the contribution of the vortex cores to $n(B)$ [20]. The parameters characterizing $G_{\mathrm{FLL}}(t)$ have to be determined by a fit procedure from the measured $G(t)$ [21]. The choice of the fit function for $G_{\mathrm{FLL}}$ faces two complications. First, the Fourier transform of $n(B)$ cannot be represented by a simple analytical function. We will follow the usual practice to approximate it by a Gaussian $\exp \left(-\sigma^{2} t^{2} / 2\right)$ where $\sigma^{2}=\gamma_{\mu}\left\langle\Delta B^{2}\right\rangle$ is assumed ( $\gamma_{\mu}$ is the gyromagnetic ratio of the $\mu^{+}$). This introduces systematic uncertainties in the evaluation of the penetration depth $\lambda$ from the fitted $\sigma$. This problem is circumvented by comparison of the fits to the experimental data with fits to simulated data [22]. The $\lambda$ values quoted below were obtained by such a procedure. The second complication arises from the inhomogeneity of the superconducting order parameter $\psi$. It is zero in the vortex core centers and rises to its maximum value $\psi_{\max }$ within the distance of a few coherence lengths from the core centers. A change of the $\mu^{+}$Knight shift $K$ due to the formation of Cooper pairs will be coupled to $\psi$. Thus also $K$ will exhibit an inhomogeneous distribution below $T_{c}$ with a value nearly equal to the value above $T_{c}$ in the vortex core centers and maximum change of $K$ between the cores. This effect will lead to an additional broadening of the field distribution experienced by the $\mu^{+}$and must be taken into account, if the shift below $T_{c}$ is of similar magnitude as the width $\left\langle\Delta B^{2}\right\rangle^{1 / 2}$. Our approach will be to separate the core (c) from the intercore (ic) regions in the fits. We approximate the spatial variation of $\psi$ by $\psi=0$ in the core and $\psi=\psi_{\max }$ in the intercore region. The volume fraction occupied by the cores is approximately $H_{\text {ext }} / H_{c 2}^{*}(T)[20,23]$. Thus we apply the two component fit function

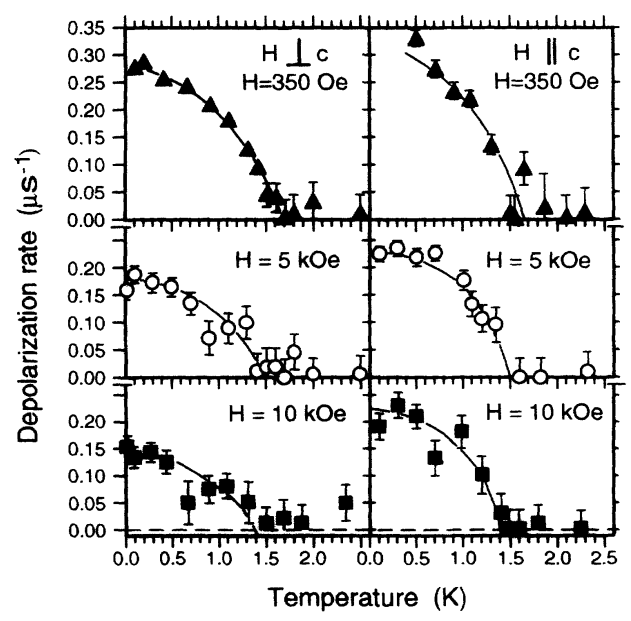

FIG. 1. Temperature dependences of the TF $\mu^{+}$depolarization rate $\sigma$ as defined in Eq. (2) in fields of $H_{\text {ext }}=350 \mathrm{Oe}$, $5 \mathrm{kOe}$, and $10 \mathrm{kOe}$, respectively. Left side: $\vec{H} \perp c$ axis; right side: $\vec{H} \|_{c}$ axis. Lines are guides to the eye.

$$
G_{\mathrm{FLL}}(t)=a_{c} \cos \left(\omega_{c} t\right)+a_{i c} \exp \left(-\frac{1}{2} \sigma^{2} t^{2}\right) \cos \left(\omega_{i c} t\right)
$$

where the ratio of the amplitudes is fixed to the theoretical value $a_{c} /\left(a_{c}+a_{i c}\right)=H_{\text {ext }} / H_{c 2}^{*}(T)$ and $\omega_{c}$ is fixed to its value above $T_{c}$ [24]. Note that the parameters relating to the intercore region now carry all the relevant information. Equations (1) and (2) yielded excellent fits to the data. The relative frequency shift in the intercore region is given by

$$
K^{*}=\omega_{i c} / \gamma_{\mu} H_{\mathrm{ext}}-1 \text {. }
$$

The observed temperature dependences of $\sigma$ and of the frequency shifts $K_{\perp, \|}^{*}$ in the intercore volume fraction are shown in Figs. 1 and 2. The shift of $T_{c}$ with field, clearly observable in Fig. 1, is in good agreement with the published value $\partial H_{c 2} / \partial T=-43 \mathrm{kOe} / \mathrm{K}$ [10]. Given the present accuracy, the behavior of $\sigma$ is practically independent of orientation and its field dependence is well in line with the behavior expected from the modified London model [20]. Comparison of the present results with simulated data (see description of the procedure above) yields for the penetration depth $\lambda_{\perp}(0)=4800 \pm$ $500 \AA$ and $\lambda_{\|}(0)=4500 \pm 500 \AA$. These values are somewhat smaller than the results from previous $\mu \mathrm{SR}$ experiments, where $\lambda(0)=6250 \pm 1250 \AA$ was found in a polycrystal [17] and $\lambda_{\|}(0)=6500 \AA$ was estimated from a measurement at $H_{\text {ext }}=1.2 \mathrm{kOe}$ [18], respectively. This discrepancy is probably due to sample quality that is reflected in different $T_{c}$ values $\left(T_{c} \simeq 1.5 \mathrm{~K}\right.$ in $\left.[17,18]\right)$. On the other hand, they agree quite well with the value $\lambda(0)=4400 \AA$ derived indirectly from $\partial H_{c 2} / \partial T$ measurements $[10,17]$ on a polycrystal $\left(T_{c}=2 \mathrm{~K}\right)$.

The present results are the first determination of the isotropic character of the penetration depth in $\mathrm{UPd}_{2} \mathrm{Al}_{3}$ and are consistent with the approximately isotropic behavior of the upper critical field $H_{c 2}[16,25,26]$ if an isotropic

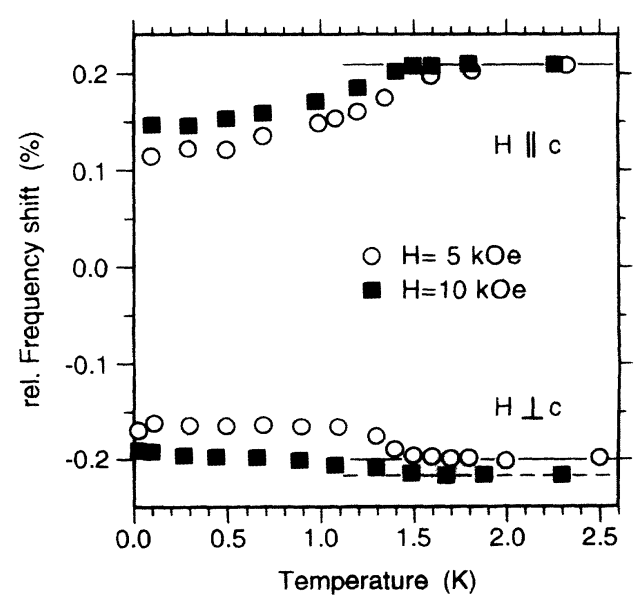

FIG. 2. Temperature dependences of the relative frequency shifts $K_{\|}^{*}$ and $K_{\perp}^{*}$ [defined in Eq. (3)] in fields of $H_{\text {ext }}=5 \mathrm{kOe}$ and $10 \mathrm{kOe}$. The data are not corrected for demagnetization fields. Lines denote the frequency shifts at $T \rightarrow T_{c}\left(T>T_{c}\right)$. Errors are of size of the symbols. 
Fermi surface is assumed. Unfortunately, due to the relatively large penetration depth and the associated rather small $\mu^{+}$depolarization rates $\sigma$, our data are not accurate enough to allow a distinction between an "exponential" and a power law behavior of $\lambda(T \rightarrow 0)$, and consequently no information on the symmetry of the superconducting order parameter can be provided.

We now discuss the $\mu^{+}$Knight shift results. The relative frequency shifts $K_{\perp, \|}^{*}$ at the axially symmetric $b$ site are given by

$$
K_{\|}^{*}=\left(A_{c}+A_{\mathrm{dip}}\right) \chi_{5 f, \|}+A_{d, L} \chi_{\text {bulk }, \|}
$$

and

$$
K_{\perp}^{*}=\left(A_{c}-\frac{1}{2} A_{\mathrm{dip}}\right) \chi_{5 f, \perp}+A_{d, L} \chi_{\text {bulk, },},
$$

where the first term is the $\mu^{+}$Knight shift $K$ and the second term arises from demagnetization and Lorentz fields. The non-5 $f$ contribution to the total shift is negligible and is thus omitted. Assuming constant hyperfine coupling $A_{c}$ and dipole coupling $A_{\text {dip }}$, the Knight shift is a direct measure of the $5 f$ susceptibility associated with the nearest $U$ neighbors of the $\mu^{+}$(or probe nucleus in NMR). The data presented in Fig. 2 are not corrected for demagnetization and Lorentz fields, which contribute about $-0.025 \%$ for $\vec{H} \| c$ and $-0.084 \%$ for $\vec{H} \perp c$ to the total shift at $2.5 \mathrm{~K}$. Since $\chi$ bulk is strongly dominated by $\chi_{5 f}$, these contributions mirror the behavior of the $5 f$ susceptibility as well. From investigations of the normal state $\mu^{+}$Knight shift (not shown) it can be concluded that the hyperfine contact coupling $A_{c}$ does not change at least down to $2.5 \mathrm{~K}$ in agreement with ${ }^{27} \mathrm{Al} \mathrm{NMR}$ findings [27]. One finds $A_{c}=1.1 \mathrm{kG} / \mu_{B}$. It is smaller than the dipole contribution $A_{\text {dip }}=3.3 \mathrm{kG} / \mu_{B}$. The anisotropy of the dipolar contribution leads to opposite signs of the total shifts $K_{\|}^{*}, K_{\perp}^{*}$ (see Fig. 2), in contrast to the ${ }^{27} \mathrm{Al} \mathrm{NMR}$ Knight shift which is dominated by the contact coupling. The field dependence of $K_{\perp}^{*}$ is in line with the known field dependence of the susceptibility [28].

Significant reductions of $K_{\perp, \|}^{*}$ are observed below $T_{c}$. The relative frequency shift change $\Delta K$ caused by the formation of Cooper pairs can be expressed as $\Delta K=K^{*}-K_{c}=\left(\omega_{i c}-\omega_{c}\right) / \gamma_{\mu} H_{\text {ext }}$. Most interesting is the fact that $\Delta K_{\|}$is negative while $\Delta K_{\perp}$ is positive. The shifts are reduced by $\left|\Delta K_{i} / K_{i}\right|=44(2) \%$ for $\vec{H} \|_{c}$ and $18(2) \%$ for $\vec{H} \perp c$ at $5 \mathrm{kOe}$, and $30(2) \%$ for $\vec{H} \| c$ and $11(2) \%$ for $\vec{H} \perp c$ at $10 \mathrm{kOe}$, respectively. These observations agree very well with the results from ${ }^{27} \mathrm{Al}$ NMR measurements [27].

A diamagnetic shift due to flux expulsion cannot account for the observed behavior, since it would be negative, independent of the orientation. The observed frequency shifts at $H_{\text {ext }}=350 \mathrm{Oe}$, however, in contrast to the shifts at $5 \mathrm{kOe}$ and $10 \mathrm{kOe}$, are dominated by the diamagnetism below $T_{c}$ and are estimated to correspond to a field of $0.8 \mathrm{Oe}$, consistent with previous results on a polycrystalline sample [17]. Thus we expect for the diamagnetic shift $\left|K_{\text {dia }}\right| \leq 100 \mathrm{ppm}$ at $5 \mathrm{kOe}$ and a much smaller value at $10 \mathrm{kOe}$. A reduction of $A_{c}$ cannot account for the observations as well. From Eqs. (4) and (5) it follows that a change of $A_{c}$ would lead to shifts of the same sign for both orientations and the magnitude of $K_{\perp}$ would be much larger than that of $K_{\|}$ (because $\chi_{\perp} \approx 3 \chi_{\|}$at $T \rightarrow 0$ ), in clear contrast to the observation. We conclude that the hyperfine coupling $A_{c}$, determined by the RKKY mechanism, is constant over the whole temperature range $0.1<T<300 \mathrm{~K}$, consistent with the observation that the magnetism is unaifected below $T_{c}$ [15]. The dipolar coupling $A_{\mathrm{dip}}$, however, is determined by the lattice geometry and is constant below $T_{c}$. Thus the only explanation for the observed Knight shift reduction is that it reflects a partial reduction of the $5 f$ susceptibility, $\chi_{5 f}$, in the superconducting state. Note that the similar interpretation of the NMR Knight shift, for which the dipolar fields are negligible, was based on the a priori assumption of a constant $A_{c}$ [27]. The $\mu \mathrm{SR}$ results, in contrast, being strongly dependent on the dipolar fields, allow a direct determination of $\chi_{5 f}$. Since the frequency shifts reflect the behavior of the $5 f$ susceptibility, namely, $\Delta K_{i}^{*} / K_{i}^{*} \propto \Delta \chi_{i} / \chi_{i}$, one can derive from the observed frequency shift reductions absolute values for the susceptibility reductions. With $\chi_{\|}\left(T \rightarrow T_{c}\right)=3.88 \times 10^{-3} \mathrm{emu} / \mathrm{mole}$ and $\chi_{\perp}\left(T \rightarrow T_{c}\right)=9.95 \times 10^{-3} \mathrm{emu} / \mathrm{mole}$ (measured at $H_{\text {ext }}=6 \mathrm{kOe}$ ), we obtain for $T \rightarrow 0 \Delta \chi_{\|}=1.7(1) \times$ $10^{-3} \mathrm{emu} / \mathrm{mole}$ and $\Delta_{\chi_{\perp}}=1.8(2) \times 10^{-3} \mathrm{emu} / \mathrm{mole}$. For $H_{\text {ext }}=10 \mathrm{kOe} \Delta \chi_{\|}=1.2(1) \times 10^{-3} \mathrm{emu} / \mathrm{mole}$ and $\Delta \chi_{\perp}=1.2(2) \times 10^{-3} \mathrm{emu} / \mathrm{mole}$ are derived. Surprisingly $\Delta \chi_{5 f}$ is approximately isotropic, in contrast to the total $5 f$ susceptibility for $T \rightarrow T_{c}$. Clearly $\Delta \chi_{5 f}$ is associated with the superconducting heavy electron system. A susceptibility reduction in the superconducting state is compatible with singlet pairing. Strong evidence for singlet pairing has been found in the previously reported pronounced paramagnetic limiting of $H_{c 2}(T)$ [17]. Since superconductivity in $\mathrm{UPd}_{3} \mathrm{Al}_{3}$ is known to show clean limit properties [17], one expects that almost all (heavy) electrons outside the vortex cores have condensed into Cooper pairs at $T \rightarrow 0$. The observed field dependence of $\Delta \chi_{5 f}$ is most probably connected with the paramagnetic limiting effect. It causes a breaking of Cooper pairs in the intercore region and hence an increase, with increasing field, of the number of unpaired electrons that contribute to the $5 f$ susceptibility. Thus $\Delta_{\chi_{5 f}} \simeq 1.7 \times 10^{-3} \mathrm{emu} /$ mole is a (lower) estimate for the total $5 f$ susceptibility connected with those electrons which carry the superconductivity. Note that the isotropy of $\Delta \chi_{5 f}$ is characteristic of an itinerant electron system.

On the other hand there is clear evidence that essentially localized $5 f$ electron states exist in $\mathrm{UPd}_{2} \mathrm{Al}_{3}$. Thus, crystal electric field effects result in a pronounced anisotropy of the magnetic susceptibility with an easy basal plane [26,28]. The residual $\left(\chi_{5 f}-\Delta_{\chi_{5 f}}\right)$ for $T \rightarrow$ 0 , which is clearly anisotropic, is associated with the local moments also responsible for the AFM ordering, that is, not affected by the superconducting transition [15]. Re- 
cently it was shown by polarized neutron scattering that in $\mathrm{UPd}_{2} \mathrm{Al}_{3}$ the magnetization density induced by an external field is essentially localized at the U ions [29]. However, from the same measurements it was concluded that at $36 \mathrm{~K}$ a fraction $\Delta \chi=2.1(4) \times 10^{-3} \mathrm{emu} / \mathrm{mole}$ of the total susceptibility is carried by more delocalized electrons. Our results imply that they correspond to electron states of $5 f$ character.

The present results lead to conclusions similar to those drawn recently from specific heat measurements under pressure [14], namely, that the electrons behave as if they were separated into two rather independent subsystems of $5 f$ character. One subsystem carries the local moment antiferromagnetism, and is connected with a strongly anisotropic susceptibility and a $20 \%$ contribution to the $T$ linear specific heat $\propto \gamma T$ [14]. The second subsystem is a more itinerant one, characterized as a "heavy electron system" by a large Sommerfeld coefficient of the specific heat $\left(\gamma=115 \mathrm{~mJ} / \mathrm{mole}^{2}\right.$ [14]) and is responsible for the superconductivity. We have shown that the latter system possesses an approximately isotropic susceptibility. Most interestingly, an estimation of the susceptibility of this electron system from $\gamma$ yields, on the basis of a simple free electron picture, $\chi=1.6 \times 10^{-3} \mathrm{emu} / \mathrm{mole}$, in close agreement with the present result.

The idea of possible coexistence of different electronic subsystems of $5 f$ character in HF compounds to our knowledge was first inferred from $\mu \mathrm{SR}$ findings in $\mathrm{UCu}_{5}$ [30]. The observation of electron states of localized as well as itinerant character may reflect the separation of $5 f$ electron spectral weight in the density of states between a dominant localized component far below the Fermi surface and itinerant degrees of freedom in the form of a narrow quasiparticle band at the Fermi surface. Taking into account this "dual" nature of $f$ electrons a phenomenological model has been proposed [31], in the framework of which the appearance of $\mathrm{HF}$ magnetism with strongly reduced ordered moments could be qualitatively explained. Whether the coexistence of local moment magnetism with independent itinerant electronic degrees of freedom can be understood in that framework is an open question. An alternative approach is to assume that the Fermi surface is divided into two regions associated with electron states of different characteristic energies $k T^{*}$ [14], implying anisotropic hybridization between $f$ and conduction electrons. Which of these approaches yields a more correct description of the electron states in $\mathrm{UPd}_{2} \mathrm{Al}_{3}$ has to be clarified by further experimental and theoretical efforts.

To conclude, we have found the London penetration depth to be essentially isotropic as well as the magnetic susceptibility reduction below $T_{c}$. The total susceptibility, however, remains strongly anisotropic. This is explained in terms of two rather independent electron subsystems of $5 f$ character, one of which is identified with the itinerant heavy quasiparticle system that is responsible for the superconductivity. The other one represents more local $5 f$ electrons and is responsible for the anti- ferromagnetic order.

We greatly acknowledge fruitful discussions with S.A.M. Mentink, J. Mydosh, M. Sigrist, and K. Ueda. We are indebted to S.Süllow (KOL, Leiden) for part of the susceptibility measurements. This work was supported in part by the SFB 252 Darmstadt/Frankfurt/Mainz.

[1] N. Grewe and F. Steglich, in Handbook on the Physics and Chemistry of Rare Earths, edited by K. A. Gschneidner, Jr. and L. Eyring (North-Holland, Amsterdam, 1991), Vol. 14, p. 343 ff.

[2] U. Sigrist and K. Ueda, Rev. Mod. Phys. 63, 239 (1991).

[3] G. M. Luke et al., Bull. Am. Phys. Soc. 34, 977 (1989); G. M. Luke et al., Hyperfine Interact. 85, 397 (1994).

[4] A. Amato, Physica (Amsterdam) 199\&200B, 91 (1994).

[5] G. Bruls et al., Phys. Rev. Lett. 72, 1754 (1994).

[6] G. Aeppli et al., Phys. Rev. Lett. 63, 676 (1989); G. M. Luke et al., Phys. Rev. Lett. 71, 1466 (1993).

[7] H.R. Ott et al., Phys. Rev. B 31, 1651 (1985); R. H. Heffner et al., Phys. Rev. Lett. 65, 2816 (1990).

[8] C. Broholm et al., Phys. Rev. B 58, 1467 (1987); E. D. Isaacs et al., Phys. Rev. 65, 3185 (1990); T.E. Mason et al., Phys. Rev. Lett. 65, 3189 (1990).

[9] C. Geibel et al., Z.Phys. B 83, 305 (1991).

[10] C. Geibel et al., Z. Phys. B 84, 1 (1991).

[11] A. Amato et al., Z. Phys. B 86, 159 (1992).

[12] A. Schröder et al., Phys. Rev. Lett. 72, 136 (1994).

[13] A. Krimmel et al., Z. Phys. B 86, 161 (1992).

[14] R. Caspary et al., Phys. Rev. Lett. 71, 2146 (1993).

[15] A. Krimmel et al., Solid State Commun. 87, 829 (1993); H. Kita et al., J. Phys. Soc. Jpn. 63, 726 (1994).

[16] N. Sato et al., J. Phys. Soc. Jpn. 61, 32 (1992).

[17] A. Amato et al., Europhys. Lett. 19, 127 (1992).

[18] Y.J. Uemura et al., Physica (Amsterdam) 186-188B, 223 (1993).

[19] $\Lambda_{0}=0.18-0.32 \mu \mathrm{s}^{-1}$ is observed, depending on the orientation and external field.

[20] E. H. Brandt, Phys. Rev. B 37, 2349 (1988).

[21] Because the shape of $n(B)$ is masked by the magnetic contribution $\Lambda_{0}, n(B)$ cannot be directly obtained from a Fourier transform of the measured $G(t)$.

[22] M. Weber et al., Phys. Rev. B 48, 13022 (1993).

[23] $H_{c 2}^{*}(T)$ is the temperature dependence of the upper critical field, extrapolated from the initial slope $\partial H_{c 2} / \partial T$ [17]. It would reflect the upper critical field in the absence of the paramagnetic limiting effect. $H_{c 2}^{*} \approx 50 \mathrm{kOe}$ for $T \rightarrow 0$.

[24] A third small signal from the sample holder exhibited a practically unshifted frequency $\omega$, which is quite different from $\omega_{c}$ (compare lines in Fig. 2) and $\omega_{i c}$, and thus could be well separated out in the data analysis..

[25] K. Gloos et al., Phys. Rev. Lett. 70, 501 (1993).

[26] C. Geibel et al., Physica (Amsterdam) 186-188B, 188 (1993).

[27] M. Kyogaku et al., J. Phys. Soc. Jpn. 62, 4016 (1993).

[28] A. Gauel et al., Phys. Rev. B 46, 5815 (1992).

[29] L. Paolasini et al., J. Phys. Condens. Matter 5, 8905 (1993).

[30] A. Schenck et al., Phys. Rev. Lett. 65, 2454 (1990).

[31] Y. Kuramoto et al., J. Phys. Soc. Jpn. 59, 2831 (1990). 